\title{
Existence of positive solution and Hyers-Ulam stability for a nonlinear singular-delay-fractional differential equation
}

\author{
Hasib Khan ${ }^{1,2^{*}}$, Thabet Abdeljawad ${ }^{3}$, Muhammad Aslam², Rahmat Ali Khan² and Aziz Khan ${ }^{4}$
}

\author{
"Correspondence: \\ hasibkhan13@yahoo.com \\ ${ }^{1}$ College of Engineering Mechanics \\ and Materials, Hohai University, \\ Nanjing, P.R. China \\ 2Department of Mathematics, \\ Shaheed Benazir Bhutto University, \\ Dir Upper, Pakistan \\ Full list of author information is \\ available at the end of the article
}

\begin{abstract}
In this article, we consider a study of a general class of nonlinear singular fractional DEs with $p$-Laplacian for the existence and uniqueness (EU) of a positive solution and the Hyers-Ulam (HU) stability. To proceed, we use classical fixed point theorem and properties of a $p$-Laplacian operator. The fractional DE is converted into an integral alternative form with the help of the Green's function. The Green's function is analyzed as regards its nature and then, with the help of a fixed point approach, the existence of a positive solution and uniqueness are studied. After the EU of a positive solution, the HU-stability and an application are considered. The suggested singular fractional DE with $\phi_{p}$ is more general than the one considered in (Khan et al. in Eur. Phys. J. Plus 133:26, 2018)
\end{abstract}

MSC: 34B82; 26A33; 45N05

Keywords: Caputo fractional derivative; Riemann-Liouville derivative; Delay-Fractional differential equations with singularity; EU of solution

\section{Introduction}

Fractional order models have attracted the attentions of researchers of various discipline, in the last two decades. In fact, fractional order models can be met with in numerous fields such as processing, control theory, signals, biology, fluid dynamics,modern physics, set theory, hydrodynamics, viscoelastic theory, computer networking and others. For detailed literature connected to these topics, we refer the reader to some famous books [2-8]. Various mathematical procedures have been considered by scientists through different research oriented aspects of fraction DEs.

Recently, some authors explored fractional DEs with singularity with the help of different mathematical techniques. For example, Bai and Qiu [9] established EU of solutions for a nonlinear singular boundary value problem (BVP) of fractional DEs with the help of the Leray-Schauder and Krasnosel'skii's fixed point theorems. They also provided some applications to illuminate the results. Agarwal et al. $[10,11]$ investigated EU for a singular fractional BVP involving the Riemann-Liouville fractional derivative. Bai and Fang [12] explored a singular coupled system of nonlinear fractional DEs for the EU of solution with the use of Leray-Schauder and Krasnoselskii's fixed point technique. Vong [13] studied fractional DEs with singularity and non-local boundary conditions by using a Schauder

(c) The Author(s) 2019. This article is distributed under the terms of the Creative Commons Attribution 4.0 International License (http://creativecommons.org/licenses/by/4.0/), which permits unrestricted use, distribution, and reproduction in any medium, provided you give appropriate credit to the original author(s) and the source, provide a link to the Creative Commons license, and indicate if changes were made. 
fixed point approach and using upper-lower solution techniques. Zhang et al. [14] investigated positive solutions of a multipoint BVP with singularity and applied the results to a specific example to illuminate the results. Khan et al. [15] studied a nonlinear singular fractional DE with singularity and $p$-Laplacian for the EU of solutions and stability analysis. They also presented an important application of their work.

Hybrid fractional DEs were studied by several authors for the EU of solutions with the help of different mathematical procedures. For instance, Kumam et al. [16] highlighted the study of EU of solutions for a coupled system of high order hybrid functional DEs using a classical fixed point approach by Krasnosel'skii. Chasreechai et al. [17] explored a hybrid fractional sum-difference initial and BVP in a Banach algebra and presented an instructive example.

Some authors worked on the EU of solutions for fractional DEs with $p$-Laplacian operator. For instance; Li [18] studied a fractional DE for the EU of positive solutions having integral boundary conditions and the nonlinear $p$-Laplacian operator. Wang [19] examined the EU of positive solutions for a class of mixed fractional BVP and $p$-Laplacian. Khan et al. [1] studied a nonlinear fractional DE with $p$-Laplacian operator for the EU of solutions and a vital application was presented. On the other hand, existence and uniqueness of delay fractional differential equations were first investigated in [20,21] one decade ago. For more existence and uniqueness results in fractional calculus one may refer to [22-25].

From the literature we note that no such investigation to p-Laplacian operator in the frame of delay fractional DEs with singularity has been considered. Therefore, inspired by the aforementioned work we investigate the following proposal for the existence of a positive solution (EPS) and stability analysis:

$$
\left\{\begin{array}{l}
\mathcal{D}^{\beta}\left[\Phi_{p}\left[\mathcal{D}^{\vartheta_{0}}\left[x(t)-y_{2}^{*}(t, x(t))\right]\right]\right]=-\mathcal{A}^{*}(t) y_{1}^{*}(t, x(t-\tau)), \\
\left.\left(\Phi_{p}\left[\mathcal{D}^{\vartheta_{0}} x(t)-y_{2}^{*}(t, x(t))\right]\right)\right|_{t=0}=0=\left.\left(\Phi_{p}\left[\mathcal{D}^{\vartheta_{0}} x(t)-y_{2}^{*}(t, x(t))\right]\right)^{\prime}\right|_{t=0}, \\
x(0)=0=x^{\prime}(1), \quad \mathcal{I}^{2-\vartheta_{0}}\left[x(t)-\left.y_{2}^{*}(t, x(t)]\right|_{t=0}=0,\right.
\end{array}\right.
$$

where $\beta \in(1,2], \vartheta_{0} \in(2,3]$ and $y_{1}^{*}, \mathcal{W}$ are continuous but singular at some points. The fractional derivatives $\mathcal{D}^{\beta}$ and $\mathcal{D}^{\vartheta_{0}}$ are taken in the Caputo sense and in the RiemannLiouville sense, respectively, $\Phi_{p}(r)=|r|^{p-2} r$ is a nonlinear $\Phi_{p}$-operator satisfying $1 / p+$ $1 / q=1$ and $\Phi_{p}^{-1}=\Phi_{q}$. By the positive solution $x(t)$ of the suggested fractional DE (1.1) we mean $x(t)>0$ for $t \in(0,1]$, satisfying (1.1). Our projected $p$-Lapacian delay fractional DEs with singularity operator $\Phi_{p}$ is more general than the problems considered in [26-28].

In this paper, we are involved in the study of EU of solutions and stability for the fractional DEs with operator $p$-Laplacian relating time and space singularity and $\tau>0$ delay. To the best of our knowledge, in the literature there is no article investigating the mentioned problem. In order to study this problem, we will change it to fractional integral form with the help of the Green function $\mathcal{G}^{\vartheta_{0}}(t, s)$. Throughout the investigation, the monotonicity properties of the Green function will be considered in the given interval $(1,2]$. Further, using fixed point theorems the EU of solutions will be demonstrated and the stability will be checked. As an application, we give and analyse an example. The reader may further consider multiplicity results using different definitions of the fractional derivatives. We take a help of in our work from [29-41]. 
Definition 1.1 The Riemann-Liouville fractional integral of a function $\psi$ of order $y_{1}>0$, $\psi:(0,+\infty) \rightarrow \mathbb{R}$, is given by

$$
\mathcal{I}^{y_{1}} \psi(t)=\frac{1}{\Gamma\left(y_{1}\right)} \int_{0}^{t}(t-s)^{y_{1}-1} \psi(s) d s,
$$

where for $\operatorname{Re}\left(y_{1}\right)>0$ we have

$$
\Gamma\left(y_{1}\right)=\int_{0}^{+\infty} e^{-s} s^{y_{1}-1} d s
$$

Definition 1.2 The fractional order derivative in the Caputo sense for a continuous function $\psi(t):(0,+\infty) \rightarrow \mathbb{R}$, is given as

$$
\mathcal{D}^{\vartheta_{0}} \psi(t)=\frac{1}{\Gamma\left(k-y_{1}\right)} \int_{0}^{t}(t-s)^{k-y_{1}-1} \psi^{(k)}(s) d s,
$$

for $k=\left[y_{1}\right]+1$, where $\left[y_{1}\right]$ is integer part of $y_{1}$, such that the integral is well defined on $(0,+\infty)$ interval.

Lemma 1.1 ([29]) For a fractional order $y_{1} \in(n-1, n], \psi \in C^{n-1}$, the following is satisfied:

$$
\mathcal{I}^{y_{1}} \mathcal{D}_{1}^{y} \psi(t)=\psi(t)+m_{0}+m_{1} t+m_{2} t^{2}+\cdots+m_{n-1} t^{n-1},
$$

for the $m_{k} \in \mathbb{R}$ for $k=0,1,2, \ldots, n-1$.

We utilize the well-known Guo-Krasnosel'skii theorem for the existence of positive solution.

Theorem $1.2([42,43])$ Consider a Banach space $\mathcal{Y}$ and let $P \in \mathcal{Y}$ be a cone. Suppose that $\mathcal{W}_{1}, \mathcal{W}_{2}$ are two bounded subsets of $\mathcal{Y}$ such that $0 \in \mathcal{W}_{1}, \overline{\mathcal{W}_{1}} \subset \mathcal{W}_{2}$, and the operator $\mathcal{F}^{*}: P \cap\left(\overline{\mathcal{W}_{2}} \backslash \mathcal{W}_{1}\right) \rightarrow P$ be continuous such that

$\left(\mathcal{N}_{1}\right)\left\|\mathcal{F}^{*} x\right\| \leq\|x\|$ if $x \in P \cap \partial \mathcal{W}_{1}$ and $\left\|\mathcal{F}^{*} x\right\| \geq\|x\|$ if $x \in P \cap \partial \mathcal{W}_{2}$, or

$\left(\mathcal{N}_{2}\right)\left\|\mathcal{F}^{*} x\right\| \geq\|x\|$ if $x \in P \cap \partial \mathcal{W}_{1}$ and $\left\|\mathcal{F}^{*} x\right\| \leq\|x\|$ if $x \in P \cap \partial \mathcal{W}_{2}$.

Then $\mathcal{F}^{*}$ has a fixed point in $P \cap\left(\overline{\mathcal{W}_{2}} \backslash \mathcal{W}_{1}\right)$.

Lemma 1.3 ([15]) Let $\Phi_{p}$ be the nonlinear $\Phi_{p}$-operator. Then

(1) for $1<p \leq 2, \gamma_{1}^{*} \gamma_{2}>0$ and $\left|\gamma_{1}^{*}\right|,\left|\gamma_{2}^{*}\right| \geq \rho>0$, then

$$
\left|\Phi_{p}\left(\gamma_{1}^{*}\right)-\Phi_{p}\left(\gamma_{2}^{*}\right)\right| \leq(p-1) \rho^{p-2}\left|\gamma_{1}^{*}-\gamma_{2}^{*}\right|
$$

(2) If $p>2$, and $\left|\gamma_{1}^{*}\right|,\left|\gamma_{2}^{*}\right| \leq \rho^{*}$, then

$$
\left|\Phi_{p}\left(\gamma_{1}^{*}\right)-\Phi_{p}\left(\gamma_{2}^{*}\right)\right| \leq(p-1) \rho^{* p-2}\left|\gamma_{1}^{*}-\gamma_{2}^{*}\right| .
$$

\section{Green function and its properties}

Theorem 2.1 For $\beta \in(1,2], \vartheta \in(2,3]$ a function $y_{1}^{*} \in C[0,1]$ satisfies (1.1) if and only if

$$
x(t)=y_{2}^{*}(t, x(t))+\int_{0}^{1} \mathcal{G}^{\vartheta_{0}}(t, s) \Phi_{q}\left(\frac{1}{\Gamma(\beta)} \int_{0}^{s}(s-\zeta)^{\beta-1} f(\zeta) y_{1}^{*}(\zeta, x(\zeta))\right) d \zeta d s,
$$


where $\mathcal{G}^{\vartheta_{0}}(t, s)$ is a Green's function given by

$$
\mathcal{G}^{\vartheta_{0}}(t, s)= \begin{cases}\frac{-\left(t-s \vartheta^{\vartheta_{0}-1}\right.}{\Gamma\left(\vartheta_{0}\right)}+\frac{t^{\vartheta_{0}-1}(1-s)^{\vartheta_{0}-2}}{\Gamma\left(\vartheta_{0}\right)} & s \leq t, \\ \frac{t^{\vartheta} 0^{-1}(1-s)^{\vartheta_{0}-2}}{\Gamma\left(\vartheta_{0}\right)}, & s \geq t .\end{cases}
$$

Proof If we apply the fractional integral operator $\mathcal{I}^{\beta}$ on (1.1) and make use of Lemma 1.1, then problem (1.1) becomes

$$
\Phi_{p}\left[\mathcal{D}^{\beta} x(t)-y_{2}^{*}(t, x(t))\right]=-\mathcal{I}^{\beta}\left[f(t) y_{1}^{*}(t, x(t-\tau))\right]+c_{1}+c_{2} t
$$

The conditions $\left(\left.\Phi_{p}\left(\mathcal{D}^{\vartheta_{0}}\left[x(t)-y_{2}^{*}(t, x(t))\right]\right)\right|_{t=0}=0=\left(\left.\Phi_{p}\left(\mathcal{D}^{\vartheta_{0}}\left[x(t)-y_{2}^{*}(t, x(t))\right]\right)^{\prime}\right|_{t=0}\right.\right.$, imply that $c_{1}=c_{2}=0$. Also, we get

$$
\Phi_{p}\left(\mathcal{D}^{\vartheta_{0}}\left[x(t)-y_{2}^{*}(t, x(t))\right]\right)=-\mathcal{I}^{\alpha}\left[f(t) y_{1}^{*}(t, x(t-\tau))\right] .
$$

From (2.4), we further have

$$
\mathcal{D}^{\vartheta_{0}}\left[x(t)-y_{2}^{*}(t, x(t))\right]=-\Phi_{q}\left(\mathcal{I}^{\beta}\left[f(t) y_{1}^{*}(t, x(t-\tau)) d t\right]\right) .
$$

Applying integral operator of fractional order $\mathcal{I}^{\vartheta_{0}}$ on (2.5) and using Lemma 1.1 again, we have

$$
\begin{aligned}
x(t)-y_{2}^{*}(t, x(t))= & -\mathcal{I}^{\vartheta_{0}}\left(\Phi_{q}\left(\mathcal{I}^{\beta}\left[f(t) y_{1}^{*}(t, x(t-\tau))\right]\right)\right) \\
& +c_{1}^{*} t^{\vartheta_{0}-1}+c_{2}^{*} t^{\vartheta_{0}-2}+c_{3}^{*} t^{\vartheta-3},
\end{aligned}
$$

where $x(0)=0$ implies that $c_{3}^{*}=0, \mathcal{I}^{2-\vartheta_{0}}\left[x(t)-\left.y_{2}^{*}(t, x(t)]\right|_{t=0}=0\right.$ implies that $c_{2}^{*}=0$ Now for $c_{1}^{*}$, using condition $x^{\prime}(1)=0$, we have

$$
c_{1}^{*}=\left.\frac{1}{\vartheta_{0}-1} \mathcal{I}^{\vartheta_{0}-1}\left(\Phi_{q}\left(\mathcal{I}^{\beta}\left[f(t) y_{1}^{*}(t, x(t-\tau))\right)\right]\right)\right|_{t=1} .
$$

Putting the values of $c_{1}^{*}$ and $c_{2}^{*}$ in (2.6), we get

$$
\begin{aligned}
x(t)= & y_{2}^{*}(t, x(t))-\mathcal{I}^{\vartheta_{0}}\left(\Phi_{q}\left(\mathcal{I}^{\beta}\left[f(t) y_{1}^{*}(t, x(t-\tau))\right)\right]\right) \\
& +\left.\frac{t^{\vartheta_{0}-1}}{\vartheta_{0}-1} \mathcal{I}^{\vartheta_{0}-1}\left(\Phi_{q}\left(\mathcal{I}^{\beta}\left[f(t) y_{1}^{*}(t, x(t-\tau))\right)\right]\right)\right|_{t=1} \\
= & y_{2}^{*}(t, x(t))+\left[-\int_{0}^{t} \frac{(t-s)^{\vartheta_{0}-1}}{\Gamma\left(\vartheta_{0}\right)}+t^{\vartheta_{0}-1} \int_{0}^{1} \frac{(1-s)^{\vartheta_{0}-1}}{\Gamma\left(\vartheta_{0}\right)}\right] \\
& \Phi_{q}\left(\frac{1}{\Gamma\left(\vartheta_{0}\right)} \int_{0}^{s}(s-\zeta)^{\beta-1}\left[f(\zeta) y_{1}^{*}(\zeta, x(\zeta-\tau))\right] d \zeta\right) d s \\
= & y_{2}^{*}(t, x(t)) \\
& +\int_{0}^{1} \mathcal{G}^{\vartheta_{0}}(t, s) \Phi_{q}\left(\frac{1}{\Gamma(\beta)} \int_{0}^{s}(s-\zeta)^{\beta-1}\left[f(t) y_{1}^{*}(\zeta, x(\zeta-\tau))\right] d \zeta\right) d s,
\end{aligned}
$$

where $\mathcal{G}^{\vartheta_{0}}(t, s)$ is well defined by $(2.2)$. 
Lemma 2.2 The function $\mathcal{G}^{\vartheta_{0}}(t, s)$ defined by Eq. (2.2), satisfies:

$\left(\mathcal{N}_{1}\right) 0<\mathcal{G}^{\vartheta_{0}}(t, s)$ for all $s, t \in(0,1)$;

$\left(\mathcal{N}_{2}\right)$ the function $\mathcal{G}^{\vartheta_{0}}(t, s)$ is increasing and $\mathcal{G}^{\vartheta_{0}}(1, s)=\max _{t \in[0,1]} \mathcal{G}^{\vartheta_{0}}(t, s)$ and

$\left(\mathcal{N}_{3}\right) \mathcal{G}^{\vartheta_{0}}(t, s) \geq t^{\vartheta-1} \max _{t \in[0,1]} \mathcal{G}^{\vartheta_{0}}(t, s)$ for $s, t \in(0,1)$.

Proof To evaluate the proof of $\left(\mathcal{N}_{1}\right)$, we assume two cases.

Case 1: For $s \leq t$, consider

$$
\begin{aligned}
\mathcal{G}^{\vartheta_{0}}(t, s) & =-\frac{(t-s)^{\vartheta_{0}-1}}{\Gamma\left(\vartheta_{0}\right)}+t^{\vartheta_{0}-1} \frac{(1-s)^{\vartheta_{0}-2}}{\Gamma\left(\vartheta_{0}\right)} \\
& =-t^{\vartheta_{0}-1} \frac{\left(1-\frac{s}{t}\right)^{\vartheta_{0}-1}}{\Gamma\left(\vartheta_{0}\right)}+t^{\vartheta_{0}-1} \frac{(1-s)^{\vartheta_{0}-2}}{\Gamma\left(\vartheta_{0}\right)} \\
& \geq-t^{\vartheta_{0}-1} \frac{(1-s)^{\vartheta_{0}-1}}{\Gamma\left(\vartheta_{0}\right)}+t^{\vartheta_{0}-1} \frac{(1-s)^{\vartheta_{0}-2}}{\Gamma\left(\vartheta_{0}\right)} \geq 0 .
\end{aligned}
$$

Case 2: For $t \leq s$, we have

$$
\mathcal{G}^{\vartheta_{0}}(t, s)=t^{\vartheta_{0}-1} \frac{(1-s)^{\vartheta_{0}-2}}{\Gamma\left(\vartheta_{0}\right)}>0
$$

With (2.9) and (2.10), it is proved that $\mathcal{G}^{\vartheta_{0}}(t, s)>0$ for all $0<s, t<1$.

For $\left(\mathcal{N}_{2}\right)$, we consider that:

Case 1 when $s \leq t$, we proceed to find

$$
\begin{aligned}
\frac{\partial}{\partial t} \mathcal{G}^{\vartheta_{0}}(t, s) & =-\left(\vartheta_{0}-1\right) \frac{(t-s)^{\vartheta_{0}-2}}{\Gamma\left(\vartheta_{0}\right)}+\left(\vartheta_{0}-1\right) t^{\vartheta_{0}-2} \frac{(1-s)^{\vartheta_{0}-2}}{\Gamma\left(\vartheta_{0}\right)} \\
& =-t^{\vartheta_{0}-2} \frac{\left(1-\frac{s}{t}\right)^{\vartheta_{0}-2}}{\Gamma\left(\vartheta_{0}-1\right)}+t^{\vartheta_{0}-2} \frac{(1-s)^{\vartheta_{0}-2}}{\Gamma\left(\vartheta_{0}-1\right)} \\
& =-t^{\vartheta_{0}-2} \frac{(1-s)^{\vartheta_{0}-2}}{\Gamma\left(\vartheta_{0}-1\right)}+t^{\vartheta_{0}-2} \frac{(1-s)^{\vartheta_{0}-2}}{\Gamma\left(\vartheta_{0}-1\right)}>0 .
\end{aligned}
$$

Case 2: For $t \leq s$, we evaluate

$$
\frac{\partial}{\partial t} \mathcal{G}^{\vartheta_{0}}(t, s)=\left(\vartheta_{0}-1\right) t^{\vartheta_{0}-2} \frac{(1-s)^{\vartheta_{0}-2}}{\Gamma\left(\vartheta_{0}\right)}>0
$$

With (2.11), (2.12), we have $\frac{\partial}{\partial t} \mathcal{G}^{\vartheta_{0}}(t, s)>0$ for $s, t \in(0,1)$. This implies that the Green's function $\mathcal{G}^{\vartheta_{0}}(t, s)$ is increasing with respect to $t$. Thus, for $t \geq s$, we arrive at

$$
\max _{t \in[0,1]} \mathcal{G}^{\vartheta_{0}}(t, s)=\frac{(1-s)^{\vartheta_{0}-2}}{\Gamma\left(\vartheta_{0}\right)}=\mathcal{G}^{\vartheta_{0}}(1, s)
$$

Similarly, for $s \geq t$, we have

$$
\max _{t \in[1,2]} \mathcal{G}^{\vartheta_{0}}(t, s)=\frac{(1-s)^{\vartheta_{0}-1}}{\Gamma\left(\vartheta_{0}-1\right)}+\frac{(1-s)^{\vartheta_{0}-2}}{\Gamma\left(\vartheta_{0}-1\right)}=\mathcal{G}^{\vartheta_{0}}(1, s)
$$

For $\left(\mathcal{N}_{3}\right)$, we presume two cases. 
Case 1: For $t \geq s$, then

$$
\begin{aligned}
\mathcal{G}^{\vartheta_{0}}(t, s) & =-\frac{(t-s)^{\vartheta_{0}-1}}{\Gamma\left(\vartheta_{0}\right)}+t^{\vartheta_{0}-1} \frac{(1-s)^{\vartheta_{0}-2}}{\Gamma\left(\vartheta_{0}\right)} \\
& \geq-\frac{t^{\vartheta_{0}-1}\left(1-\frac{s}{t}\right)^{\vartheta_{0}-1}}{\Gamma\left(\vartheta_{0}\right)}+\frac{t^{\vartheta_{0}-1}(1-s)^{\vartheta_{0}-2}}{\Gamma\left(\vartheta_{0}\right)} \\
& \geq-\frac{t^{\vartheta_{0}-1}(1-s)^{\vartheta_{0}-1}}{\Gamma\left(\vartheta_{0}\right)}+\frac{t^{\vartheta_{0}-1}(1-s)^{\vartheta_{0}-2}}{\Gamma\left(\vartheta_{0}\right)} \geq 0 .
\end{aligned}
$$

Case 2: For $s \geq t$, then

$$
\mathcal{G}^{\vartheta_{0}}(t, s)=t^{\vartheta_{0}-1} \frac{(1-s)^{\vartheta_{0}-2}}{\Gamma\left(\vartheta_{0}\right)}=t^{\vartheta_{0}-1} \max _{t \in[0,1]} \mathcal{G}^{\vartheta_{0}}(t, s)=t^{\vartheta_{0}-1} \mathcal{G}^{\vartheta_{0}}(1, s)
$$

By (2.15) and (2.16), the proof of $\left(\mathcal{N}_{3}\right)$ is accomplished.

\section{Existence results}

Consider a Banach space $\mathcal{Y}=\mathcal{C}[0,1]$ with a norm $\|x\|=\max _{t \in[0,1]}\{|x(t)|: x \in \mathcal{Y}\}$ and let $P$ be a cone containing non-negative functions in the space $\mathcal{Y}$, where $P=\{x \in \mathcal{Y}: x(t) \geq$ $\left.t^{\epsilon}\|x\|, t \in[0,1]\right\}$. Let $\mathcal{W}(r)=\{x \in P:\|x\|<r\}, \partial \mathcal{W}(r)=\{x \in P:\|x\|=r\}$.

With the help of Theorem 2.1, an alternate form of (1.1) is

$$
\begin{aligned}
x(t)= & y_{2}^{*}(t, x(t)) \\
& +\int_{0}^{1} \mathcal{G}^{\vartheta_{0}}(t, s) \Phi_{q}\left(\frac{1}{\Gamma(\beta)} \int_{0}^{s}(s-\zeta)^{\beta-1}\left[\mathcal{A}^{*}(t) y_{1}^{*}(t, x(t-\tau)] d \zeta d s\right) .\right.
\end{aligned}
$$

Define $\mathcal{F}^{*}: P \backslash\{0\} \rightarrow \mathcal{Y}$ by

$$
\begin{aligned}
\mathcal{F}^{*} x(t)= & y_{2}^{*}(t, x(t)) \\
& +\int_{0}^{1} \mathcal{G}^{\vartheta_{0}}(t, s) \Phi_{q}\left(\int_{0}^{1}(s-\zeta)^{\beta}(s, \zeta)\left[\mathcal{A}^{*}(t) y_{1}^{*}(t, x(t-\tau)] d t\right) d s .\right.
\end{aligned}
$$

With the help of Theorem 2.1, the solution of (1.1), i.e., $x(t)$ is equivalent to a fixed point of $\mathcal{F}$, that is,

$$
x(t)=\mathcal{F}^{*} x(t) .
$$

Presume the following conditions:

$\left(\mathcal{P}_{1}\right) y^{*}:((0,1) \times(0,+\infty)) \rightarrow[0,+\infty)$ is continuous.

$\left(\mathcal{P}_{2}\right) A:(0,1) \rightarrow[0,+\infty)$ is discontinuous on $(0,1)$ and non vanishing and $\|A\|=$ $\max _{t \in[0,1]}\left|\mathcal{A}^{*}(t)\right|<+\infty$.

$\left(\mathcal{P}_{3}\right)$ For $a_{1}, a_{2}, \chi_{1}^{*}, \chi_{2}^{*}$ positive constants and $k_{1} \in[0,1]$, the functions $y_{1}^{*}, y_{2}^{*}$ satisfy

$$
\begin{gathered}
\left|y_{1}^{*}(t, x(t))\right| \leq \Phi_{p}\left(a_{1}|x(t)|^{k_{1}}+\chi_{1}^{*}\right) \\
\left|y_{2}^{*}(t, x(t))\right| \leq \phi_{p}\left(a_{2}|x(t)|^{k_{2}}+\chi_{2}^{*}\right) .
\end{gathered}
$$


$\left(\mathcal{P}_{4}\right)$ For a constant value $\lambda_{1}, \lambda_{2}>0$ and $u, v \in \mathcal{Y}$, the functions $y_{1}^{*}, y_{2}^{*}$ satisfy

$$
\begin{aligned}
& \left|y_{1}^{*}(t, x(t))-y_{1}^{*}(t, v(t))\right| \leq \lambda_{1}|x(t)-v(t)|, \\
& \left|y_{2}^{*}(t, x(t-\tau))-y_{2}^{*}(t, v(t-\tau))\right| \leq \lambda_{2}|x(t)-v(t)| .
\end{aligned}
$$

Theorem 3.1 Assume that conditions $\left(\mathcal{P}_{1}\right)-\left(\mathcal{P}_{3}\right)$ are satisfied. Then $\mathcal{F}^{*}$ is completely continuous operator.

Proof For any $x \in \overline{\mathcal{W}\left(r_{2}\right)} \backslash \mathcal{W}\left(r_{1}\right)$, we have from Lemma 2.2 and (3.2) that

$$
\begin{aligned}
\mathcal{F}^{*} x(t)= & y_{2}^{*}(t, x(t))+\int_{0}^{1} \mathcal{G}^{\vartheta_{0}}(t, s) \Phi_{q}\left(\frac{1}{\Gamma\left(\vartheta_{0}\right)} \int_{0}^{s}(s-\zeta)^{\vartheta_{0}-1}\left[\mathcal{A}^{*}(t) y_{1}^{*}(t, x(t-\tau)] d t\right) d s\right. \\
\leq & \int_{0}^{1} \mathcal{G}^{\vartheta_{0}}(1, s) \Phi_{q}\left(\frac{1}{\Gamma\left(\vartheta_{0}\right)} \int_{0}^{s}(s-\zeta)^{\vartheta_{0}-1}\left[A(t) y_{1}^{*}(t, x(t-\tau)] d t\right) d s\right. \\
\mathcal{F}^{*} x(t)= & y_{2}^{*}(t, x(t))+\int_{0}^{1} \mathcal{G}^{\vartheta_{0}}(t, s) \Phi_{q}\left(\frac{1}{\Gamma(\beta)} \int_{0}^{s}(s-\zeta)^{\vartheta_{0}-1}\left[\mathcal{A}^{*}(t) y_{1}^{*}(t, x(t-\tau)] d t\right) d s\right. \\
\geq & y_{2}^{*}(t, x(t)) \\
& +t^{\vartheta_{0}-1} \int_{0}^{1} \mathcal{G}^{\vartheta_{0}}(1, s) \Phi_{q}\left(\frac{1}{\Gamma(\beta)} \int_{0}^{s}(s-\zeta)^{\beta-1}\left[\mathcal{A}^{*}(t) y_{1}^{*}(t, x(t-\tau)] d t\right) d s .\right.
\end{aligned}
$$

With help of (3.4) and (3.5), we proceed to find

$$
\mathcal{F}^{*} x(t) \geq t^{\vartheta_{0}-1}\left\|\mathcal{F}^{*} x(t)\right\|, \quad t \in[0,1]
$$

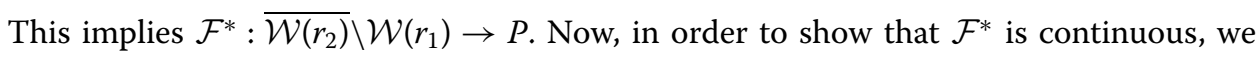
prove $\left\|\mathcal{F}^{*}\left(x_{n}\right)-\mathcal{F}^{*}(x)\right\| \rightarrow 0$ as $n \rightarrow \infty$, given by

$$
\begin{aligned}
\mid \mathcal{F}^{*} x_{n}(t)-\mathcal{F}^{*} x(t) \| & \\
=\mid & \mid y_{2}^{*}\left(t, x_{n}(t)\right)-y_{2}^{*}(t, x(t)) \\
& +\left(\int_{0}^{1} \mathcal{G}^{\vartheta_{0}}(t, s) \Phi_{q}\right)\left(\frac{1}{\Gamma(\beta)} \int_{0}^{s}(s-\zeta)^{\beta-1}\left[\mathcal{A}^{*}(t) y_{1}^{*}\left(t, x_{n}(t-\tau)\right)\right] d t\right) d s \\
& \left.\quad-\int_{0}^{1} \mathcal{G}^{\vartheta_{0}}(t, s) \Phi_{q}\left(\frac{1}{\Gamma(\beta)} \int_{0}^{s}(s-\zeta)^{\beta-1}\left[\mathcal{A}^{*}(t) y_{1}^{*}(t, x(t-\tau))\right] d t\right) d s\right) \mid \\
\leq & \int_{0}^{1}\left|\mathcal{G}^{\vartheta_{0}}(t, s)\right| \mid \Phi_{q}\left(\frac{1}{\Gamma(\beta)} \int_{0}^{s}(s-\zeta)^{\beta-1}\left[\mathcal{A}^{*}(t) y_{1}^{*}\left(t, x_{n}(t-\tau)\right)\right] d t\right) d s \\
& -\Phi_{q}\left(\frac{1}{\Gamma(\beta)} \int_{0}^{s}(s-\zeta)^{\beta-1}\left[\mathcal{A}^{*}(t) y_{1}^{*}(t, x(t-\tau))\right] d t\right) \mid d s .
\end{aligned}
$$

With the help of (3.7), and continuity of $y_{1}^{*}, y_{2}^{*}$ we have $\left|\mathcal{F}^{*} x_{n}(t)-\mathcal{F}^{*} x(t)\right| \rightarrow 0$ as $n \rightarrow+\infty$, which shows that $\mathcal{F}^{*}$ is continuous. Now, for the uniformly boundedness of $\mathcal{F}^{*}$, by (3.2) 
and $\left(P_{2}\right)$, we get

$$
\begin{aligned}
\left|\mathcal{F}^{*} x(t)\right|= & \mid y_{2}^{*}(t, x(t)) \\
& \left.+\int_{0}^{1} \mathcal{G}^{\vartheta_{0}}(t, s) \Phi_{q}\left(\frac{1}{\Gamma(\beta)} \int_{0}^{s}(s-\zeta)^{\beta-1}\left[\mathcal{A}^{*}(t) y_{1}^{*}(t, x(t)-\tau)\right)\right] d t\right) d s \mid \\
= & \mid y_{2}^{*}(t, x(t)) \\
& +\int_{0}^{1}\left|\mathcal{G}^{\vartheta_{0}}(t, s)\right| \Phi_{q}\left(\frac{1}{\Gamma(\beta)} \int_{0}^{s}(s-\zeta)^{\beta-1}\left[\left|\mathcal{A}^{*}(t) y_{1}^{*}(t, x(t-\tau))\right|\right] d t\right) d s \\
\leq & \mid y_{2}^{*}(t, x(t)) \\
& +\int_{0}^{1}\left|\mathcal{G}^{\vartheta_{0}}(1, s)\right| \Phi_{q}\left(\frac{1}{\Gamma(\beta)} \int_{0}^{s}(s-\zeta)^{\beta-1}\|A\| \Phi_{p}\left(a_{1}\|x\|^{k_{1}}+\chi^{*} y *\right) d t\right) d s \\
\leq & \left(\frac{2}{\Gamma\left(\vartheta_{0}+1\right)}+\frac{1}{\Gamma\left(\vartheta_{0}\right)}\right)\left[\frac{1}{\Gamma(\beta+1)}\right]^{q-1}\|\mathcal{A}\|^{q-1}\left(a_{1}\|x\|^{k_{1}}+\chi_{1}^{*}\right) \\
& +a_{2}\|x\|_{2}^{k}+\chi^{*} \\
= & \Delta_{1}\|\mathcal{A}\|^{q-1}\left(a_{1}\|x\|+\chi^{*}\right)+a_{2}\|x\|_{2}^{k}+\chi_{2}^{*},
\end{aligned}
$$

where $\Delta_{1}=\left(\frac{1}{\Gamma\left(\vartheta_{0}+1\right)}+\frac{1}{\left(\vartheta_{0}-1\right) \Gamma\left(\vartheta_{0}\right)}\right)\left[\frac{1}{\Gamma\left(\vartheta_{0}+1\right)}\right]^{q-1}$. By (3.8), the operator $\mathcal{F}^{*}: \overline{\mathcal{W}\left(r_{2}\right)} \backslash \mathcal{W}\left(r_{1}\right)$ is uniformly bounded. Now for the equicontinuity of the operator $\mathcal{F}^{*}$, by $\left(\mathcal{P}_{3}\right)$, Theorem 2.1 and (3.2), for any $t_{1}, t_{2} \in[0,1]$, we have

$$
\begin{aligned}
\left|\mathcal{F}^{*} x\left(t_{1}\right)-\mathcal{F}^{*} x\left(t_{2}\right)\right| & \\
\leq & \mid y_{2}^{*}\left(t_{1}, x\left(t_{1}\right)-y_{2}^{*}\left(t_{2}, x\left(t_{2}\right) \mid\right.\right. \\
& \left.+\mid \int_{0}^{1} \mathcal{G}^{\vartheta_{0}}\left(t_{1}, s\right) \Phi_{q}\left(\frac{1}{\Gamma\left(\vartheta_{0}\right)} \int_{0}^{s}(s-\zeta)^{\vartheta_{0}-1}\left[\mathcal{A}^{*}(t) y_{1}^{*}(t, x(t-\tau))\right] d t\right) d s\right) \\
& \quad-\int_{0}^{1} \mathcal{G}^{\vartheta_{0}}\left(t_{2}, s\right) \Phi_{q}\left(\frac{1}{\Gamma\left(\vartheta_{0}\right)} \int_{0}^{s}(s-\zeta)^{\vartheta_{0}-1}\left[\mathcal{A}^{*}(t) y_{1}^{*}(t, x(t-\tau))\right] d t\right) d s \mid \\
\leq & \int_{0}^{1}\left|\mathcal{G}^{\vartheta_{0}}\left(t_{1}, s\right)-\mathcal{G}^{\vartheta_{0}}\left(t_{2}, s\right)\right| \\
& \times \Phi_{q}\left(\frac{1}{\Gamma\left(\vartheta_{0}\right)} \int_{0}^{s}(s-\zeta)^{\vartheta_{0}-1}\|A\| \Phi_{p}\left(a_{1}\|x\|^{k_{1}}+\chi_{1}^{*}\right) d \zeta\right) d s \\
\leq & \left(\frac{\left|t_{1}^{\vartheta_{0}}-t_{2}^{\vartheta_{0}}\right|}{\Gamma\left(\vartheta_{0}+1\right)}+\frac{\left|t^{\vartheta_{0}-1}-t^{\vartheta_{0}-1}\right|}{\Gamma\left(\vartheta_{0}\right)}\right)\left[\frac{1}{\Gamma(\beta+1)}\right]^{q-1}\|\mathcal{A}\|^{q-1}\left(a_{1}\|x\|^{k_{1}}+\chi_{1}^{*}\right) .
\end{aligned}
$$

As $t_{1} \rightarrow t_{2}$, we find that (3.9) goes to zero. Hence $\mathcal{F}^{*}\left(\overline{\mathcal{W}\left(r_{2}\right)} \backslash \mathcal{W}\left(r_{1}\right)\right)$ is an equicontinuous operator and by the Arzela-Ascoli theorem $\mathcal{F}^{*}\left(\overline{\mathcal{W}\left(r_{2}\right)} \backslash \mathcal{W}\left(r_{1}\right)\right)$ is compact. This proves that $\mathcal{F}^{*}$ is compact in $\overline{\mathcal{W}\left(r_{2}\right)} \backslash \mathcal{W}\left(r_{1}\right)$. Consequently; $\mathcal{F}^{*}: \overline{\mathcal{W}\left(r_{2}\right)} \backslash \mathcal{W}\left(r_{1}\right) \rightarrow P$ is completely continuous.

Here, we define height for $y^{*}(t, x(t))$ for $r>0$, and

$$
\left\{\begin{array}{l}
\Phi_{\max }(t, r)=\max _{t \in(0,1)}\left\{y_{1}^{*}(t, x(t-\tau)): t^{\vartheta_{0}-1} r \leq x \leq r\right\} \\
\Phi_{\min }(t, r)=\min _{t \in(0,1)}\left\{y_{1}^{*}(t, x(t-\tau)): t^{\vartheta_{0}-1} r \leq x \leq r\right\}
\end{array}\right.
$$


Theorem 3.2 Assume that $\left(\mathcal{P}_{1}\right)-\left(\mathcal{P}_{3}\right)$ hold true and there exist $a, b \in \mathbb{R}^{+}$such that

$\left(\mathcal{W}_{1}\right) a \leq\left\|y_{2}^{*}(t, x(t))\right\|+\int_{0}^{1} \mathcal{G}^{\vartheta_{0}}(1, s) \Phi_{q}\left(\frac{1}{\Gamma\left(\vartheta_{0}\right)} \int_{0}^{s}(s-\zeta)^{\vartheta_{0}-1} \Phi_{\min }(\zeta, a) d t\right) d s<+\infty$ and $\left\|y_{2}^{*}(t, x(t))\right\|+\int_{0}^{1} \mathcal{G}^{\vartheta_{0}}(1, s) \Phi_{q}\left(\frac{1}{\Gamma(\beta)} \int_{0}^{s}(s-\zeta)^{\beta-1}\left[f(\zeta) \Phi_{\max }(\zeta, b)\right] d \zeta\right) d s \leq b$, or

$\left(\mathcal{W}_{2}\right)\left\|y_{2}^{*}(t, x(t))\right\|+\int_{0}^{1} \mathcal{G}^{\vartheta_{0}}(1, s) \Phi_{q}\left(\frac{1}{\Gamma\left(\vartheta_{0}\right)} \int_{0}^{s}(s-\zeta)^{\vartheta_{0}-1}\left[\Omega(\zeta) \Phi_{\max }(\zeta, a)\right] d t\right) d s<a$ and $b \leq$ $\left\|y_{2}^{*}(t, x(t))\right\|+\int_{0}^{1} \mathcal{G}^{\vartheta_{0}}(1, s) \Phi_{q}\left(\frac{1}{\Gamma\left(\vartheta_{0}\right)} \int_{0}^{s}(s-\zeta)^{\vartheta_{0}-1}\left[\Omega(\zeta) \Phi_{\min }(\zeta, b)\right] d t\right) d s<+\infty$,

is satisfied. Then the fractional DE with operator $\Phi_{p}(1.1)$ has a positive solution $x \in P$ and $a \leq\|x\| \leq b$.

Proof With no loss of generalization, consider the case $\left(\mathcal{W}_{1}\right)$. If $x \in \partial \mathcal{W}(a)$, then we have $\|x\|=a$ and $t^{\vartheta_{0}-1} a \leq x(t) \leq a, t \in[0,1]$. With the help of (3.10), for $t \in(0,1)$, we have $\Phi_{\min }(t, u) \leq y_{1}^{*}(t, x(t-\tau))$, which implies

$$
\begin{aligned}
\left\|\mathcal{F}^{*} x(t)\right\| & =\max _{t \in[0,1]} \int_{0}^{1} \mathcal{G}^{\vartheta_{0}}(t, s) \Phi_{q}\left(\frac{1}{\Gamma(\beta)} \int_{0}^{s}(s-\zeta)^{\beta-1}\left[\mathcal{A}^{*}(t) y^{*}(t, x(t-\tau))\right] d t\right) d s \\
& \left.\geq t^{\vartheta_{0}-1} \int_{0}^{1} \mathcal{G}^{\vartheta_{0}}(1, s) \Phi_{q}\left(\frac{1}{\Gamma(\beta)} \int_{0}^{s}(s-\zeta)^{\beta-1}\left[f(\zeta) y^{*}(t, x(t-\tau))\right] d t\right]\right) d s \\
& \geq \int_{0}^{1} \mathcal{G}^{\vartheta_{0}}(1, s) \Phi_{q}\left(\frac{1}{\Gamma(\beta)} \int_{0}^{s}(s-\zeta)^{\beta-1}\left[\mathcal{A}^{*}(t) \Phi_{\min }(t, a)\right] d t\right) d s \geq a \\
& =\|x\| .
\end{aligned}
$$

If $x(t) \in \partial \mathcal{W}(b)$, then $\|x\|=b$ and $t^{\vartheta_{0}-1} b \leq u \leq b$, for $0 \leq t \leq 1$. By (3.10), we get $\Phi_{\max }(t, u) \geq y^{*}(t, x(t-\tau))$, and we proceed to find

$$
\begin{aligned}
\left\|\mathcal{F}^{*} x(t)\right\|= & \max _{t \in[0,1]}\left[y_{2}^{*}(t, x(t))\right. \\
& +\int_{0}^{1} \mathcal{G}^{\vartheta_{0}}(t, s) \Phi_{q}\left(\frac{1}{\Gamma(\beta)} \int_{0}^{s}(s-\zeta)^{\beta-1}\left[\mathcal{A}^{*}(t) y^{*}(t, x(t-\tau))\right] d t\right) d s \\
\leq & t^{\vartheta_{0}-1} \int_{0}^{1} \mathcal{G}^{\vartheta_{0}}(1, s) \Phi_{q}\left(\frac{1}{\Gamma(\beta)} \int_{0}^{s}(s-\zeta)^{\beta-1}\left[\mathcal{A}^{*}(t) y^{*}(t, x(t-\tau))\right] d t\right) d s \\
\leq & \| y_{2}^{*}(t, x(t) \| \\
& +\int_{0}^{1} \mathcal{G}^{\vartheta_{0}}(1, s) \Phi_{q}\left(\frac{1}{\Gamma(\beta)} \int_{0}^{s}(s-\zeta)^{\beta-1}\left[f(\zeta) \Phi_{\max }(\zeta, b)\right] d \zeta\right) d s \\
\leq & b=\|x\| .
\end{aligned}
$$

By Lemma 1.2, $\mathcal{F}^{*}$ has a fixed point say $x \in \overline{\mathcal{W}(b)} \backslash \mathcal{W}(a)$. Thus, we have $a \leq\left\|x^{*}\right\| \leq b$, which by Lemma 2.2 and Theorem 2.1 implies $x^{*}(t) \geq t^{\vartheta_{0}-1}\left\|x^{*}\right\| \geq a t^{\vartheta_{0}-1}>0$, for $t \in(0,1)$. Thus $x^{*}$ is an increasing positive solution

$$
\begin{aligned}
\frac{\partial}{\partial t} x^{*}(t)= & \frac{\partial}{\partial t} \mathcal{F}^{*} x(t) \\
= & \frac{\partial}{\partial t} y_{2}^{*}(t, x(t)) \\
& +\int_{0}^{1} \frac{\partial}{\partial t} \mathcal{G}^{\vartheta_{0}}(t, s) \Phi_{q}\left(\frac{1}{\Gamma(\beta)} \int_{0}^{s}(s-\zeta)^{\beta-1}\left[\mathcal{A}^{*}(t) y^{*}\left(t, x^{*}(t-\tau)\right)\right] d t\right) d s
\end{aligned}
$$$$
>0 \text {. }
$$ 


\section{Stability analysis}

Here, a stability analysis is presented for the fractional DE with nonlinear $\Phi_{p}$-operator of the problem (1.1). The following definition is given on the basis of [1,28] and related literature.

Definition 4.1 We say that (3.1) is Hyers-Ulam stable if for every $\lambda>0$, there exists a constant value $\mathcal{D}^{*}>0$, such that the following holds true:

if

$$
\begin{aligned}
& \left|x(t)-y_{2}^{*}(t, x(t))-\int_{0}^{1} \mathcal{G}^{\vartheta_{0}}(t, s) \Phi_{q}\left(\frac{1}{\Gamma(\beta)} \int_{0}^{s}(s-\zeta)^{\beta-1}\left[\mathcal{A}^{*}(t) y_{1}^{*}(\zeta, x(\zeta-\tau))\right] d \zeta\right) d s\right| \\
& \quad \leq \lambda
\end{aligned}
$$

there exists $u(t)$ satisfying that

$$
u(t)=\int_{0}^{1} \mathcal{G}^{\vartheta_{0}}(t, s) \Phi_{q}\left(\frac{1}{\Gamma(\beta)} \int_{0}^{s}(s-\zeta)^{\beta-1}\left[\mathcal{A}^{*}(\zeta) y_{1}^{*}(\zeta, u(\zeta-\tau))\right] d \zeta\right) d s
$$

such that

$$
|x(t)-u(t)| \leq \mathcal{L}^{*} \lambda^{*}
$$

Theorem 4.1 The singular fractional $D E$ with delay and $\Phi_{p}$-operator, the problem (1.1) is Hyers-Ulam stable provided that $\left(\mathcal{P}_{1}\right),\left(\mathcal{P}_{2}\right)$ and $\left(\mathcal{P}_{4}\right)$ are satisfied.

Proof By Theorem 3.2 and Definition 4.1, we assume that $x(t)$ is a solution of the fractional DE with delay (3.1) and $y(t)$ is an approximate solution and satisfying (4.2). Then we have

$$
\begin{aligned}
\mid x(t) & -u(t) \mid \\
= & \left|y_{2}^{*}(t, x(t))-y_{2}^{*}(t, u(t))\right| \\
& +\mid \int_{0}^{1} \mathcal{G}^{\vartheta_{0}}(t, s) \Phi_{q}\left(\frac{1}{\Gamma(\beta)} \int_{0}^{s}(s-\zeta)^{\beta-1}\left[\mathcal{A}^{*}(t) y_{1}^{*}(t, x(t-\tau))\right] d t\right) d s \\
& \quad-\int_{0}^{1} \mathcal{G}^{\vartheta_{0}}(t, s) \Phi_{q}\left(\frac{1}{\Gamma(\beta)} \int_{0}^{s}(s-\zeta)^{\beta-1}\left[\mathcal{A}^{*}(t) y_{1}^{*}(t, u(\zeta-\tau))\right] d t\right) d s \mid \\
\leq & (p-1) \rho^{p-2}\left\|\mathcal{A}^{*}\right\|^{q-1}\left(\int_{0}^{1}\left|\mathcal{G}^{\vartheta_{0}}(t, s)\right| \mid \Phi_{q}\left(\frac{1}{\Gamma(\beta)} \int_{0}^{s}(s-\zeta)^{\beta-1}\left[y_{1}^{*}(t, x(t-\tau))\right] d t\right) d s\right. \\
& -\Phi_{q}\left(\frac{1}{\Gamma(\beta)} \int_{0}^{s}(s-\zeta)^{\beta-1}\left[y_{1}^{*}(t, u(t-\tau))\right] d t\right) d s \mid \\
\leq & (p-1) \rho^{p-2} \lambda_{y_{1}^{*}}\left(\frac{1}{\Gamma\left(\vartheta_{0}+1\right)}+\frac{1}{\left(\vartheta_{0}-1\right) \Gamma\left(\vartheta_{0}\right)}\right)\left[\frac{1}{\Gamma(\beta+1)}\right]^{q-1} \\
& \times\|\mathcal{A}\|^{q-1}\|x-u\|,
\end{aligned}
$$

where $\mathcal{L}^{*}=(p-1) \rho^{p-2} \lambda_{y_{1}^{*}}\left(\frac{1}{\Gamma\left(\vartheta_{0}+1\right)}+\frac{1}{\left(\vartheta_{0}-1\right) \Gamma\left(\vartheta_{0}\right)}\right)\left[\frac{1}{\Gamma(\beta+1)}\right]^{q-1}\|\mathcal{A}\|^{q-1}$. Hence (4.4) is HyersUlam stable. Consequently, the singular fractional DE with delay and operator $\Phi_{p}(1.1)$ is Hyers-Ulam stable. 


\section{Illustrative example}

In this section, an application of the results which have proved in Sects. 3 and 4, is provided.

Example 5.1 For $\psi_{1}(t, x(t-\tau))=x^{5}(t)+\frac{-\tau+1}{\sqrt{x(t)}}, t \in[0,1], p=3, q=1.5, \beta=\vartheta_{0}=1.5, \tau=0.3$, $\mathcal{A}(t)=\frac{1}{\sqrt{1-t}}, y_{1}^{*}(t, x(t))=x^{5}(t)+\frac{1}{5 \sqrt{x(t)}}, y_{2}^{*}(t, x(t))=0.002$, clearly $\mathcal{A} \in C((0,1),[0,+\infty)), y_{1}^{*} \in$ $C\left((0,1) \times(0,+\infty),[0,+\infty)\right.$. Presume a singular fractional DE with $\Phi_{p}$-operator:

$$
\left\{\begin{array}{l}
\mathcal{D}^{\beta}\left[\Phi_{p}\left[\mathcal{D}^{\vartheta_{0}}\left(x(t)-y_{2}^{*}(t, x(t))\right)\right]\right]+\left[\frac{1-\tau}{\sqrt{x(t)}}+x^{5}(t)\right] \frac{1}{\sqrt{1-t}}=0, \\
\left.\left(\Phi_{p}\left[\mathcal{D}^{\beta}\left(x(t)-y_{2}^{*}(t, x(t))\right)\right]\right)\right|_{t=0}=0=\left(\left.\phi_{p}\left(\mathcal{D}^{\vartheta_{0}}\left(x(t)-y_{2}^{*}(t, x(t))\right)\right)^{\prime}\right|_{t=0},\right. \\
\quad x(1)=0=x^{\prime}(0) .
\end{array}\right.
$$

We consider

$$
\begin{aligned}
& \Phi_{\max }(t, r)=\max \left\{x^{5}+\frac{1-\tau}{x^{\frac{3}{35}}}: t^{\frac{5}{3}} r \leq x \leq r\right\} \leq r^{5}+\frac{1}{5} \frac{0.7}{t^{\frac{1}{7}} r^{\frac{3}{35}}}, \\
& \Phi_{\min }(t, r)=\min \left\{x^{5}+\frac{1-\tau}{x^{\frac{3}{35}}}: t^{\frac{5}{3}} r \leq x \leq r\right\} \geq t^{5} r^{3}+\frac{1}{5 r^{\frac{3}{35}}},
\end{aligned}
$$

as height functions and

$$
y_{2}^{*}(t, x(t))= \begin{cases}0 & \text { if } t=0 \\ 0.002 & \text { if } t \in(0,1) \\ 0 & \text { if } t=1\end{cases}
$$

Then, for $t \in(0,1)$, we have

$$
\begin{aligned}
0.002+\int_{0}^{1} \mathcal{G}^{\vartheta_{0}}(1, s) \Phi_{q}\left(\frac{1}{\Gamma(\beta)} \int_{0}^{s}(s-\zeta)^{\beta-1}\left[\mathcal{A}(\zeta) \Phi_{\max }(\zeta, b)\right] d \zeta\right) d s \\
=0.002+\int_{0}^{1} \mathcal{G}^{\vartheta_{0}}(1, s) \Phi_{q}\left(\frac{1}{\Gamma(\beta)} \int_{0}^{s}(s-\zeta)^{\beta-1}\left[\mathcal{A}(\zeta) \Phi_{\max }(\zeta, 1)\right] d \zeta\right) d s \\
\leq 0.002+\int_{0}^{1} \mathcal{G}^{\vartheta_{0}}(1, s)\left(\frac{(s-\zeta)^{\beta}}{\Gamma(\beta+1)} \frac{1}{\sqrt{1-\zeta}}\left(1+\frac{1}{5 \zeta^{\frac{1}{7}}}\right)\right)^{1.5} d \zeta d s \\
=0.535426<1 \\
0.002+\int_{0}^{1} \mathcal{G}^{\vartheta_{0}}(t, s) \Phi_{q}\left(\frac{1}{\Gamma(\beta)} \int_{0}^{s}(s-\zeta)^{\beta-1}\left[\mathcal{A}(\zeta) \psi_{\min }(\zeta, a)\right] d \zeta\right) d s \\
=0.002+\int_{0}^{1} \mathcal{G}^{\vartheta_{0}}(1, s) \Phi_{q}\left(\frac{1}{\Gamma(\beta)} \int_{0}^{s}(s-\zeta)^{\beta-1}\left[\mathcal{A}(\zeta) \psi_{\min }\left(\zeta, \frac{1}{1000}\right)\right] d \zeta\right) d s \\
\geq 0.002 \\
\quad+\int_{0}^{1} \mathcal{G}^{\vartheta_{0}}(1, s) \Phi_{q}\left(\frac{1}{\Gamma(\beta)} \int_{0}^{s}(s-\zeta)^{\beta-1}\left[\frac{1}{\sqrt{1-\zeta}}\left(\zeta^{5} \frac{1}{1000^{3}}+\frac{1000^{\frac{1}{3}}}{35}\right)\right] d \zeta\right) d s \\
=0.262296>\frac{1}{1000} .
\end{aligned}
$$

With the help of Theorem 3.2, (5.1) has a solution $u *$ and satisfies $\frac{1}{1000} \leq\left\|x^{*}\right\| \leq 1$. 


\section{Conclusion}

In this paper we have considered a general class of hybrid fractional DEs (1.1) involving time and space singularities and the nonlinear operator $\phi_{p}$ for the EU of solutions and $\mathrm{HU}$ stability. For these objectives, we utilized the classical results and obtained an alternate integral formulation of the singular fractional DE (1.1). The integral equation is based on the Green's function. The Green's function was examined and proved a positive increasing function. Then with the help of fixed point approach, the existence and uniqueness of solutions were evaluated and then HU-stability was also explored. A comprehensive example was studied to deduce the applicability of the results. We suggest the reader to re-consider the problem for multiplicity and exponential stability. Also, it would be of interest to consider the existing results of this article for fractional differential operators of nonsingular kernels. The recent articles [44, 45], with the formulation of Green's type functions, are recommended in this direction.

\section{Acknowledgements}

The second author would like to thank Prince Sultan University for funding this work through research group Nonlinear Analysis Methods in Applied Mathematics (NAMAM) group number RG-DES-2017-01-17.

\section{Funding}

Not applicable.

\section{Competing interests}

The authors have no conflict of interests regarding the publication of this paper.

\section{Authors' contributions}

All the authors have equally made contributions to this paper. All authors read and approved the final manuscript.

\section{Author details}

${ }^{1}$ College of Engineering Mechanics and Materials, Hohai University, Nanjing, P.R. China. ${ }^{2}$ Department of Mathematics, Shaheed Benazir Bhutto University, Dir Upper, Pakistan. ${ }^{3}$ Department of Mathematics and General Sciences, Prince Sultan University, Riyadh, Saudi Arabia. ${ }^{4}$ Department of Mathematics, University of Peshawar, Peshawar, Pakistan.

\section{Publisher's Note}

Springer Nature remains neutral with regard to jurisdictional claims in published maps and institutional affiliations.

Received: 8 February 2019 Accepted: 4 March 2019 Published online: 12 March 2019

\section{References}

1. Khan, A., Syam, M.I., Zada, A., Khan, H.: Stability analysis of nonlinear fractional differential equations with Caputo and Riemann-Liouville derivatives. Eur. Phys. J. Plus 133, 26 (2018). https://doi.org/10.1140/epjp/i2018-12119-6

2. Samko, S.G., Kilbas, A.A., Marichev, O.I.: Fractional Integrals and Derivatives: Theory and Applications. Gordon \& Breach, Yverdon (1993)

3. Podlubny, I.: Fractional Differential Equations. Academic Press, New York (1999)

4. Hilfer, R. (ed.): Application of Fractional Calculus in Physics. World Scientific, Singapore (2000)

5. Coronel-Escamilla, A., Gómez-Aguilar, J., Baleanu, D., Córdova-Fraga, T., Escobar-Jiménez, R., Olivares-Peregrino, V., Qurashi, M.: Bateman-Feshbach Tikochinsky and Caldirola-Kanai oscillators with new fractional differentiation. Entropy 19(2), 55 (2017)

6. Yépez-Martínez, H., Gómez-Aguilar, F., Sosa, I.O., Reyes, J.M., Torres-Jiménez, J.: The Feng's first integral method applied to the nonlinear mKdV space-time fractional partial differential equation. Rev. Mex. Fis. 62(4), 310-316 (2016)

7. Atangana, A., Gómez-Aguilar, J.F.: A new derivative with normal distribution kernel: theory, methods and applications. Phys. A, Stat. Mech. Appl. 476, 1-4 (2017)

8. Atangana, A., Gómez-Aguilar, J.F.: Hyperchaotic behaviour obtained via a nonlocal operator with exponential decay and Mittag-Leffler laws. Chaos Solitons Fractals 102, 285-294 (2017)

9. Bai, Z., Qiu, T.: Existence of positive solution for singular fractional differential equation. Appl. Math. Comput. 215(7), 2761-2767 (2009)

10. Agarwal, R.P., O'Regan, D., Stanek, S.: Positive solutions for Dirichlet problems of singular nonlinear fractional differential equations. J. Math. Anal. Appl. 371(1), 57-68 (2010)

11. Agarwal, R.P., O'Regan, D., Stanek, S.: Positive solutions for mixed problems of singular fractional differential equations. Math. Nachr. 285(1), 27-41 (2012)

12. Bai, C.Z., Fang, J.X.: The existence of a positive solution for a singular coupled system of nonlinear fractional differential equations. Appl. Math. Comput. 150(3), 611-621 (2004)

13. Vong, S.: Positive solutions of singular fractional differential equations with integral boundary conditions. Math. Comput. Model. 57(5-6), 1053-1059 (2013) 
14. Pu, R., Zhang, X., Cui, Y., Li, P., Wang, W.: Positive solutions for singular semipositone fractional differential equation subject to multipoint boundary conditions. J. Funct. Spaces 2017, Article ID 5892616 (2017)

15. Khan, H., Chen, W., Sun, H.: Analysis of positive solution and Hyers-Ulam stability for a class of singular fractional differential equations with p-Laplacian in Banach space. Math. Methods Appl. Sci. 41(9), 3430-3440 (2018)

16. Kumam, W., Zada, M.B., Shah, K., Khan, R.A.: Investigating a coupled hybrid system of nonlinear fractional differential equations. Discrete Dyn. Nat. Soc. 2018, Article ID 5937572 (2018)

17. Chasreechai, S., Sitthiwirattham, T.: Existence results of initial value problems for hybrid fractional sum-difference equations. Discrete Dyn. Nat. Soc. 2018, Article ID 5268528 (2018)

18. Li, Y.: Existence of positive solutions for fractional differential equation involving integral boundary conditions with p-Laplacian operator. Adv. Differ. Equ. 2017(1), 135 (2017)

19. Wang, Y.: Existence and nonexistence of positive solutions for mixed fractional boundary value problem with parameter and $p$-Laplacian operator. J. Funct. Spaces 2018, Article ID 1462825 (2018)

20. Abdeljawad, T.A., Jarad, F., Baleanu, D.: On the existence and the uniqueness theorem for fractional differential equations with bounded delay within Caputo derivatives. Sci. China Ser. A, Math. 51(10), 1775-1786 (2008)

21. Abdeljawad, T., Baleanu, D., Jarad, F.: Existence and uniqueness theorem for a class of delay differential equations with left and right Caputo fractional derivatives. J. Math. Phys. 49, 083507 (2008)

22. Abdeljawad, T., Alzabut, J.: On Riemann-Liouville fractional $q$-difference equations and their application to retarded logistic type model. Math. Methods Appl. Sci. 41(18), 8953-8962 (2018)

23. Jarad, F., Abdeljawad, T., Hammouch, Z.: On a class of ordinary differential equations in the frame of Atangana-Baleanu fractional derivative. Chaos Solitons Fractals 117, 16-20 (2018)

24. Abdeljawad, T., Al-Mdallal, Q.M., Jarad, F.: Fractional logistic models in the frame of fractional operators generated by conformable derivatives. Chaos Solitons Fractals 119, 94-101 (2019)

25. Gambo, Y.Y., Ameen, R., Jarad, F., Abdeljawad, T.: Existence and uniqueness of solutions to fractional differential equations in the frame of generalized Caputo fractional derivatives. Adv. Differ. Equ. 2018, 134 (2018)

26. Shen, T., Liu, W., Shen, X.: Existence and uniqueness of solutions for several BVPs of fractional differential equations with $p$-Laplacian operator. Mediterr. J. Math. 13(6), 4623-4637 (2016)

27. Jafari, H., Baleanu, D., Khan, H., Khan, R.A., Khan, A.: Existence criterion for the solutions of fractional order $p$-Laplacian boundary value problems. Bound. Value Probl. 2015(1), 164 (2015). https://doi.org/10.1186/s13661-015-0425-2

28. Khan, H., Li, Y., Chen, W., Baleanu, D., Khan, A.: Existence theorems and Hyers-Ulam stability for a coupled system of fractional differential equations with p-Laplacian operator. Bound. Value Probl. 2017, Article ID 157 (2017). https://doi.org/10.1186/s13661-017-0878-6

29. Kilbas, A.A., Srivastava, H.M., Trujillo, J.J.: Theory and Applications of Fractional Differential Equations, vol. 24 North-Holland, Amsterdam (2006)

30. Khan, A., Li, Y., Shah, K., Khan, T.S.: On coupled p-Laplacian fractional differential equations with nonlinear boundary conditions. Complexity 2017, Article ID 8197610 (2017)

31. Vong, S.: Positive solutions of singular fractional differential equations with integral boundary conditions. Math. Comput. Model. 57, 1053-1059 (2013)

32. Hai, D.D.: Existence of positive solutions for periodic boundary value problem with sign-changing Green's function. Positivity 22(5), 1269-1279 (2018)

33. Dogan, A.: Positive solutions of nonlinear multi-point boundary value problems. Positivity 22(5), 1387-1402 (2018)

34. Bouchelaghem, F., Ardjouni, A., Djoudi, A.: Existence of positive solutions of delay dynamic equations. Positivity 21(4), 1483-1493 (2017)

35. Khan, H., Gómez-Aguilar, J.F., Khan, A., Khan, T.S.: Stability analysis for fractional order advection-reaction diffusion system. Phys. A, Stat. Mech. Appl. 521, 737-751 (2019)

36. Khan, H., Tunç, C., Baleanu, D., Khan, A., Alkhazzan, A.: Inequalities for n-class of functions using the Saigo fractional integral operator. Rev. R. Acad. Cienc. Exactas Fís. Nat., Ser. A Mat. 2019, 1-4 (2019)

37. Khan, H., Khan, A., Abdeljawad, T., Alkhazzan, A.: Existence results in Banach space for a nonlinear impulsive system. Adv. Differ. Equ. 2019(1), 18 (2019)

38. Jiang, W., Qiu, J., Yang, C.: The existence of solutions for fractional differential equations with $p$-Laplacian at resonance. Chaos 27, $032102(2017)$

39. Deng, W., Li, C., Lu, J.: Stability analysis of linear fractional differential system with multiple time delays. Nonlinear Dyn. 48, 409-416 (2007). https://doi.org/10.1007/s11071-006-9094-0

40. Jafari, H., Baleanu, D., Khan, H., Khan, R.A., Khan, A.: Existence criterion for the solution of fractional order $p$-Laplacian boundary value problem. Bound. Value Probl. 2015, 164 (2015)

41. Jafari, H., Jassim, H.K., Qurashi, M., Baleanu, D.: On the existence and uniqueness of solutions for local fractional differential equations. Entropy 18, 420 (2016). https://doi.org/10.3390/e18110420

42. Guo, D.J., Lakshmikantham, V.: Nonlinear Problems in Abstract Cones. Notes and Reports in Mathematics in Science and Engineering, vol. 5. Academic Press, Boston (1988)

43. Krasnosel'skii, M.A.: Positive Solutions of Operator Equations. Noordhoff, Groningen (1964)

44. Abdeljawad, T.: A Lyapunov type inequality for fractional operators with nonsingular Mittag-Leffler kernel. J. Inequal. Appl. 2017, 130 (2017). https://doi.org/10.1186/s13660-017-1400-5

45. Abdeljawad, T.: Fractional operators with exponential kernels and a Lyapunov type inequality. Adv. Differ. Equ. 2017(1), $313(2017)$

46. Qiao, Y., Zhou, Z.: Existence of positive solutions of singular fractional differential equations with infinite-point boundary conditions. Adv. Differ. Equ. 2017, 8 (2017)

47. Lakoud, A.G., Ashyralyev, A.: Positive solutions for a system of fractional differential equations with nonlocal integral boundary conditions. Differ. Equ. Dyn. Syst. 25(4), 519-526 (2017) 